\title{
Hadroproduction of the $\chi_{1}$ and $\chi_{2}$ States of Charmonium in $800 \mathrm{GeV} / \mathrm{c}$ Proton-Silicon Interactions
}

\author{
T. Alexopoulos ${ }^{18}$, L. Antoniazzi ${ }^{11}$, M. Arenton ${ }^{17}$, H.C. Ballagh ${ }^{1}$, H. Bingham ${ }^{1}$, A. Blankman ${ }^{12}$, M. Block ${ }^{10}$, \\ A. Boden ${ }^{2}$, G. Bonomi ${ }^{11}$, Z.L. Cao ${ }^{17}$, T.Y. Chen ${ }^{9}$, K. Clark $^{15}$, D. Cline ${ }^{2}$, S. Conetti ${ }^{17}$, M. Cooper ${ }^{16}$, G. Corti ${ }^{17}$, \\ B. $\mathrm{Cox}^{17, \dagger}$, P. Creti $^{7}$, E.C. Dukes ${ }^{17}$, C. Durandet ${ }^{18}$, V. Elia ${ }^{7}$, A.R. Erwin ${ }^{18}$, L. Fortney ${ }^{4}$, V. Golovatyuk ${ }^{7}$, \\ E. Gorini ${ }^{7}$, F. Grancagnolo ${ }^{7}$, K. Hagan ${ }^{17}$, M. Haire ${ }^{13}$, P. Hanlet ${ }^{17}$, M. He ${ }^{14}$, G. Introzzi ${ }^{11}$, M. Jenkins ${ }^{15}$, D. Judd ${ }^{13}$, \\ W. Kononenko ${ }^{12}$, W. Kowald ${ }^{4}$, K. Lau ${ }^{6}$, A. Ledovskoy ${ }^{17}$, G. Liguori ${ }^{11}$, J. Lys ${ }^{1}$, P.O. Mazur ${ }^{5}$, A.P. McManus ${ }^{17}$, \\ S. Misawa ${ }^{1}$, G.H. $\mathrm{Mo}^{6}$, C.T. Murphy ${ }^{5}$, K. Nelson ${ }^{17}$, V. Pogosyan $^{17}$, S. Ramachandran ${ }^{2}$, J. Rhoades ${ }^{2}$, W. Selove ${ }^{12}$, \\ R.P. Smith ${ }^{5}$, L. Spiegel ${ }^{5}$, J.G. Sun ${ }^{17}$, S. Tokar ${ }^{3}$, P. Torre ${ }^{11}$, J. Trischuk ${ }^{8}$, L. Turnbull ${ }^{13}$, D.E. Wagoner ${ }^{13}$, \\ C.R. Wang ${ }^{14}$, C. Wei ${ }^{14}$, W. Yang ${ }^{5}$, N. Yao ${ }^{9}$, N.J. Zhang ${ }^{14}$, and B.T. Zou ${ }^{4}$
}

(E771 Collaboration)

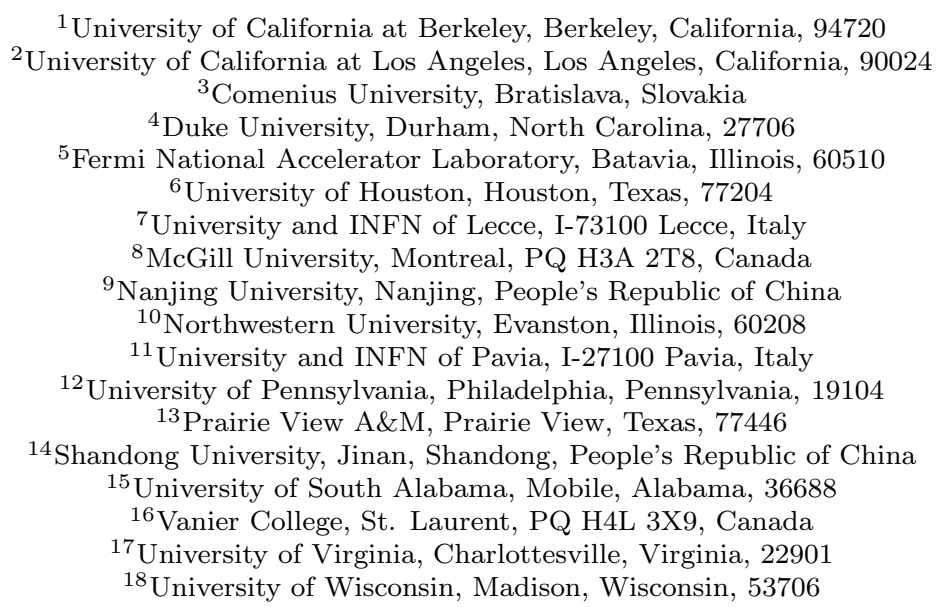

The cross sections for the hadroproduction of the $\chi_{1}$ and $\chi_{2}$ states of charmonium in proton-silicon collisions at $\sqrt{s}=38.8 \mathrm{GeV}$ have been measured in Fermilab fixed target Experiment 771. The $\chi$ states were observed via their radiative decay to $J / \psi \gamma$, where the photon converted to $e^{+} e^{-}$in the material of the spectrometer. The measured values for the $\chi_{1}$ and $\chi_{2}$ cross sections for $x_{F}>0$ are $263 \pm 69$ (stat) \pm 32 (syst) and $498 \pm 143$ (stat) \pm 67 (syst) nb per nucleon respectively. The resulting $\sigma\left(\chi_{1}\right) / \sigma\left(\chi_{2}\right)$ ratio of $0.53 \pm 0.20$ (stat) \pm 0.07 (syst), although somewhat larger than most theoretical expectations, can be accomodated by the latest theoretical estimates.

PACS numbers: 13.85.Ni, 13.85.Qk, 14.40.Gx, 25.40.Ve

Charmonium hadroproduction has provided interesting challenges to the understanding of QCD. Early attempts to describe the formation of a $c \bar{c}$ bound state, according to the color evaporation [1] or color singlet [2] models, did not provide a satisfactory description of the available data. More recently, the Non-Relativistic QCD Factorization Approach [3], which incorporates in a more rigorous fashion some of the features of the previous models, has provided a more successful description of the process. For any model, a rather crucial test has been the prediction of the relative rate of production for different charmonium states. In particular, when dealing with proton-induced processes, the absence of quark annihilation diagrams and the suppression in Leading Order of $\chi_{1}$ production gluonic diagrams implies rather small values, typically less than $10 \%$, for the ratio of $\chi_{1}$ to $\chi_{2}$ production [4. The measurement presented here represents a significant contribution to the available data, since it is the first observation of cleanly resolved $\chi_{1}$ and $\chi_{2}$ states in a proton-induced fixed target experiment.

The FNAL E771 experiment utilized a largeacceptance spectrometer [5] to measure several processes containing muons in the final state. Protons of $800 \mathrm{GeV} / \mathrm{c}$ momentum were transported by the Fermilab Proton West beam line to the High Intensity Laboratory, where they hit a $24 \mathrm{~mm}$ thick silicon target. Operating at a beam intensity of $\approx 3.6 \times 10^{7}$ protons per spillsecond, the experiment accumulated a total of $6.4 \times 10^{11}$ p-Si interactions. The incoming proton beam trajectory and flux were measured by a six plane silicon detector station. The 0.26 radiation length target was composed of twelve $2 \mathrm{~mm}$ silicon foils separated by $4 \mathrm{~mm}$. The target was followed by a microvertex detector consisting of fourteen $300 \mu \mathrm{m}$ thick silicon planes that, while not used in the analysis presented here, contributed an additional $0.045 \mathrm{X}_{0}$ to the target region radiation length.

The spectrometer's tracking system consisted of seven multi-wire proportional chambers and three drift cham- 
bers upstream plus three drift chambers and six combination drift/pad/strip chambers downstream of a dipole analysis magnet which provided an $821 \mathrm{MeV} / \mathrm{c} p_{t}$ kick in the horizontal plane. Downstream of the wire chamber system, an electromagnetic calorimeter consisting of an active converter and 396 scintillating glass and lead glass blocks was used for electron/positron identification. The final element of the spectrometer, a set of three planes of resistive plate counters (RPC's) segmented into 512 readout pads and sandwiched between layers of steel and concrete absorbers, provided muon identification. The material in the absorber walls represented an energy loss of $10 \mathrm{GeV}$ in the central region and $6 \mathrm{GeV}$ in the outer region of the detector for the incident muons.

A dimuon trigger [6] selected events with a $J / \psi$ in the final state via the decay $J / \psi \rightarrow \mu^{+} \mu^{-}$. A trigger muon was defined as the triple coincidence of the $O R$ of $2 \times 2$ pads in the first RPC plane and the $O R$ of $6 \times 6$ pads in the second and third RPC planes in projective arrangements. A dimuon trigger was defined as two such triple coincidences. The trigger reduced the $1.9 \mathrm{MHz}$ interaction rate by a factor of $\approx 10^{4}$, selecting approximately $1.3 \times 10^{8}$ dimuon events to be written to tape.

The seed for muon track reconstruction was provided by the RPC triple coincidences. The roads formed by the pads involved in the coincidences were projected into the rear chamber set, identifying a region in which to search for candidate muon tracks. Muon tracks reconstructed in the rear chamber set were then matched with tracks found in the front chamber set by requiring a good front-rear linking $\chi^{2}$. Muon pairs were required to come from a common vertex by applying a cut to their distance of closest approach. About fifty thousand dimuon events survived the reconstruction process, quality cuts, and vertex cuts.

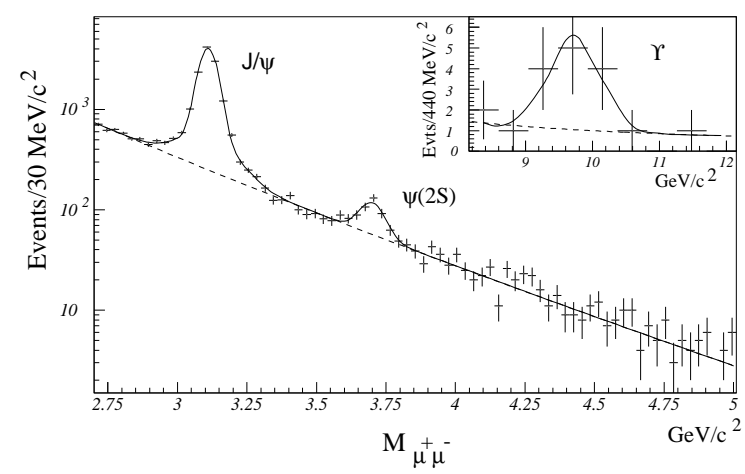

FIG. 1. Opposite sign dimuon mass spectrum. The solid line is a fit to the signal plus background and the dashed line is a fit to the background only. Inset shows the $\Upsilon$ signal.

Figure 1 shows the resulting dimuon mass spectrum containing peaks corresponding to the $J / \psi, \psi(2 S)$, and
$\Upsilon$ (inset). Superimposed on the dimuon mass spectrum is a fit to the data obtained with the sum of two Gaussians for the $J / \psi$ peak, a single Gaussian for the $\psi(2 S)$, and the form $\frac{a}{m_{\mu \mu}^{3}} \exp \left(-b m_{\mu \mu}\right)$ for the continuum background. The two Gaussians fit to the $J / \psi$ peak is a good approximation (as confirmed by Monte Carlo) to a non-constant mass resolution, caused by the confusion associated with increases in hit density near the beam region. The number of $J / \psi$ 's and $\psi(2 S)$ 's after background subtraction was $11,660 \pm 139$ and $218 \pm 24$, respectively $[7]$.

Events in a window of $\pm 100 \mathrm{MeV} / \mathrm{c}^{2}$ around the $J / \psi$ mass were refit varying the muon momenta within measurement errors, with the constraint that the invariant mass of the pair be equal to $J / \psi$ mass. The resulting dimuon event sample was then inspected to search for $e^{+} e^{-}$pairs that might be the result of conversions of photons from $\chi \rightarrow J / \psi \gamma$ decays. Dimuon events which contained pairs of tracks matching the topology of a $\gamma \rightarrow e^{+} e^{-}$conversion in the target region - collinear before the magnet in both bend and non-bend projections, collinear in the non-bend plane and coplanar in the bend plane after the magnet - were then designated as $\chi$ decay candidates.

All electron/positron pair candidates were required to satisfy additional conditions. At least one of the two track candidates was required to be associated with an energy deposition in the calorimeter consistent with an electro-magnetic shower. In addition, the total transverse momentum of the $e^{+} e^{-}$pair in the rear of the magnet was required to be zero (within the resolution of the spectrometer) relative to the common $e^{+} e^{-}$trajectory in front of the magnet. To quantify how well a pair fitted the $\gamma \rightarrow e^{+} e^{-}$hypothesis, a $\chi^{2}$ was formed,

$$
\chi^{2}=\frac{\left(a_{x 1}-a_{x 2}\right)^{2}}{\sigma_{a x 1}^{2}+\sigma_{a x 2}^{2}}+\frac{\left(a_{y 1}-a_{y 2}\right)^{2}}{\sigma_{a y 1}^{2}+\sigma_{a y 2}^{2}}+\frac{\left(b_{y 1}-b_{y 2}\right)^{2}}{\sigma_{b y 1}^{2}+\sigma_{b y 2}^{2}}
$$

where $a_{x 1}$ and $a_{x 2}$ are the electron and positron track intercepts at the magnet in the bend plane, $a_{y 1}$ and $a_{y 2}$ the track intercepts in the non-bend plane, $b_{y 1}$ and $b_{y 2}$ the track slopes in the non-bend plane, and the $\sigma$ 's are the measurement errors on these quantities. The electron/positron candidate with the smallest $\chi^{2}$ in a given event was designated as a photon conversion candidate. Additional cuts requiring a good $\chi^{2}$, the transverse momentum of the parent photon to be between 250 and 700 $\mathrm{MeV} / \mathrm{c}$, and the invariant mass squared of the $e^{+} e^{-}$pair to be less than $3000\left(\mathrm{MeV} / \mathrm{c}^{2}\right)^{2}$ were applied to maximize signal to background in the final sample of events containing both a $J / \psi$ and a photon conversion.

The $J / \psi e^{+} e^{-}$invariant mass shown in Fig. 2 was calculated using the electron and positron momenta obtained from the tracking system. Clear $\chi_{1}$ and $\chi_{2}$ signals can be seen. The background to the $\chi_{1}$ and $\chi_{2}$ was well-described by uncorrelated $e^{+} e^{-}$and $J / \psi$ combinations: the solid line of Fig. 2 was obtained by fitting 
two Gaussians plus a polynomial background. The polynoimial background was obtained by fitting the mass dis-

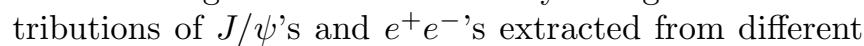
events. The numbers of $\chi_{1}$ and $\chi_{2}$ obtained from the fit are $33 \pm 9$ and $33 \pm 10$, respectively. The fitted width is $5.2 \pm 2.0 \mathrm{MeV} / \mathrm{c}^{2}$ for both the $\chi_{1}$ and $\chi_{2}$ peaks.

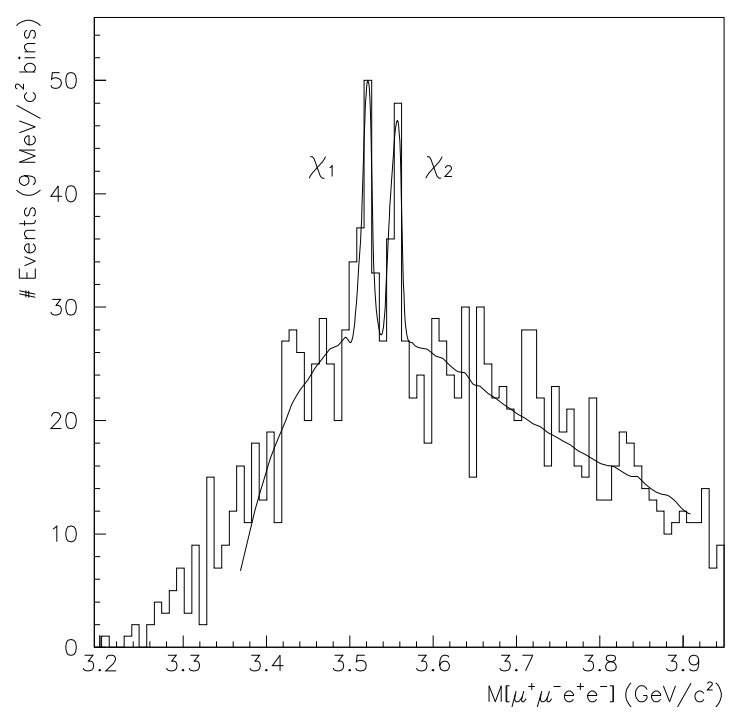

FIG. 2. $J / \psi e^{+} e^{-}$invariant mass. The solid line shows a fit to a polynomial background plus two Gaussians of equal width.

To determine the total cross section for $\chi_{1}$ and $\chi_{2}$ production, the overall acceptance times efficiency for photon conversion and for electron/positron acceptance and recontruction efficiency had to be determined. To accomplish this, a Monte Carlo sample of $\chi \rightarrow J / \psi \gamma$, $J / \psi \rightarrow \mu^{+} \mu^{-}$decays was generated using Pythia [8]. The photon and the muons were then propagated through a GEANT [9] simulation of the E771 detector, including $\gamma$ conversion, scattering, bremsstrahlung and $\mathrm{dE} / \mathrm{dx}$. Hits from the Monte Carlo tracks obtained by this prescription were then inserted into actual dimuon trigger events to simulate realistically backgrounds and losses in pattern recognition due to confusion from noise hits and other tracks. Measured detector efficiencies were also applied to the inserted hits. These hybrid Monte Carlo and data events were analyzed in a manner identical to the data in order to determine acceptances and tracking efficiencies.

Rather than attempting to simulate the electromagnetic calorimeter response to $e^{ \pm}$in detail in a Monte Carlo, the efficiency of matching an electron or positron candidate to a shower in the calorimeter was determined using a large sample of electron/positron pairs from photon conversions in minimum bias events. A sample of $e^{+} e^{-}$pairs with kinematics similar to those of the $\chi$ $e^{+} e^{-}$pairs was collected using very tight cuts to ensure an $e^{+} e^{-}$identity. This sample was then subjected to the same constraints as those applied in the $\chi$ analysis. The resulting overall acceptance times efficiency (inclusive of conversion probability) for photons from $\chi$ decay was determined to be $(8.25 \pm 0.4) \times 10^{-3}$.

Using the $\gamma \rightarrow e^{+} e^{-}$acceptance and efficiency, the measured branching ratios for $\chi_{1}$ and $\chi_{2}$ into $J / \psi \gamma[10]$, the measured $J / \psi \mathrm{pN}$ forward cross section [7] at $\sqrt{s}=38.8 \mathrm{GeV}$ and the number of observed $\chi_{1}, \chi_{2}$ and $J / \psi \rightarrow \mu^{+} \mu^{-}$, the absolute $\chi_{1}$ and $\chi_{2}$ cross sections for $x_{F}>0$ were calculated to be $\sigma\left(\chi_{1}\right)=263 \pm 69$ (stat) \pm 32 (syst) $\mathrm{nb} /$ nucleon and $\sigma\left(\chi_{2}\right)=498 \pm 143$ (stat) \pm 67 (syst) nb/nucleon, respectively. The main contributions to the systematic errors came from the error on the $J / \psi$ cross section $(9 \%)$, the uncertainty in the knowledge of the cut efficiencies (5\%) and the errors on the branching ratios for $\chi_{1}(6 \%)$ and $\chi_{2}(8 \%)$.

Using the production cross sections for $\chi_{1}$ and $\chi_{2}$, the ratio of the $\chi_{1}$ to $\chi_{2}$ production cross sections was determined to be $\sigma\left(\chi_{1}\right) / \sigma\left(\chi_{2}\right)=0.53 \pm 0.20$ (stat) \pm 0.07 (syst). Combining this result with the two previous measurements of $\chi$ production by a proton beam [1], we have computed the world average (shown in Fig. 3) to be $\sigma\left(\chi_{1}\right) / \sigma\left(\chi_{2}\right)=0.31 \pm 0.14$. This figure is consistent with the latest NRQCD estimates of $\approx 0.3[12]$, where $\chi_{1}$ production was boosted by the inclusion of higher order terms in the velocity expansion [13].

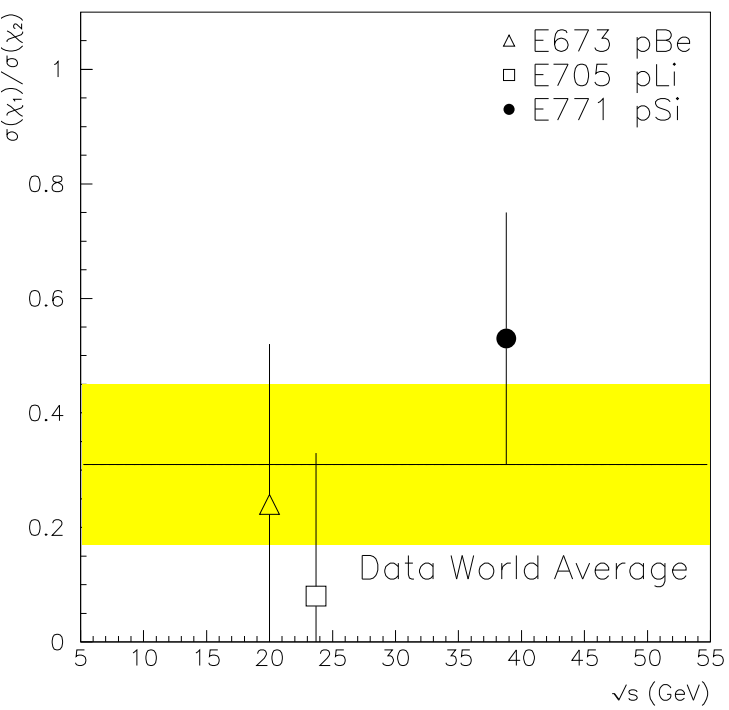

FIG. 3. $\sigma\left(\chi_{1}\right) / \sigma\left(\chi_{2}\right)$ in proton-nucleon interactions. Results from this experiment (E771) are shown, together with the two existing results and the world average with $1 \sigma$ errors.

Finally, the energy dependence of the combined $\chi_{1}$ and $\chi_{2}$ production near threshold was compared to the corresponding quantity for $J / \psi$ production. In Ref. [14] data on $J / \psi$ production from seventeen different $\mathrm{pN}$ ex- 
periments over a large range of center-of-mass energy, $\sqrt{s} \approx 8$ to $52 \mathrm{GeV}$, were fit as a function of $\sqrt{s}$. The $J / \psi$ production data near threshold was well represented by the function $\sigma(\sqrt{s})_{J / \psi}=\sigma_{0}\left(1-M_{J / \psi} / \sqrt{s}\right)^{\beta}$, with $\sigma_{0}=1.0 \pm 0.1 \mu \mathrm{b} /$ nucleon and $\beta=11.8 \pm 0.5$. To check whether $\chi$ production has similar dynamics as $J / \psi$ production, the sum of the $\chi$ cross sections have been fit to a similar parameterization with $\beta$ fixed to the $J / \psi$ value and $M_{\chi}$ replacing $M_{J / \psi}$. The result of the fit, shown in Fig. 4, shows the similarity of the $J / \psi$ threshold production parametrization to the threshold behavior of the combined $\chi$ state cross sections. The fit yields $\sigma_{0}=2.3 \pm 0.4 \mu \mathrm{b} /$ nucleon for the asymptotic $\sigma(\chi)$ cross section.

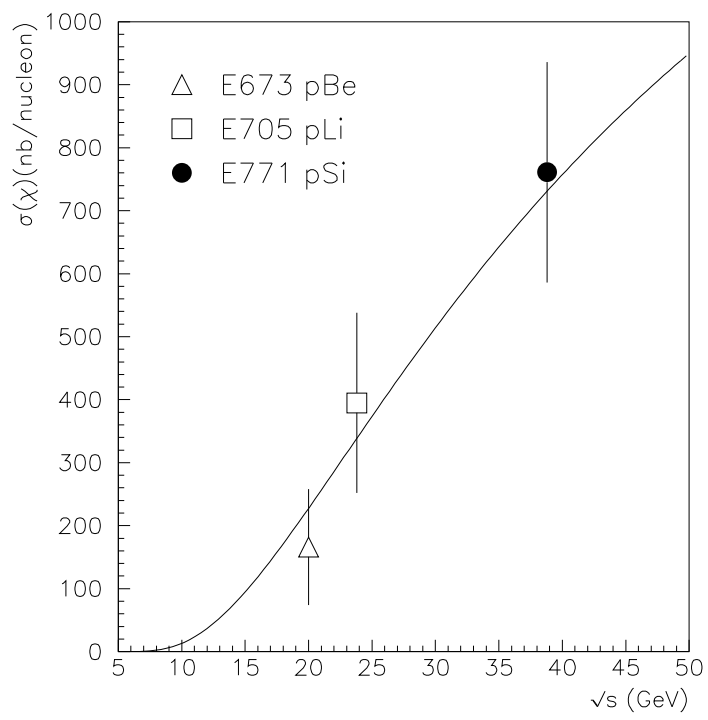

FIG. 4. $\sigma(\chi)$ vs. $\sqrt{s}$ in proton-nucleon interactions. Superimposed on the data is a fit to a threshold production parameterization described in the text; here $\sigma(\chi)$ is the sum of the $\chi_{1}$ and $\chi_{2}$ cross sections where the E673 data point has been obtained from the published $\chi_{1}$ cross section and ratio of $\chi_{2}$ to $\chi_{1}$

We wish to thank Fermilab, the U.S. Department of Energy, the National Science Foundation, the Istituto Nazionale di Fisica Nucleare of Italy, the Natural Science and Engineering Research Council of Canada, the Institute for Particle and Nuclear Physics of the Commonwealth of Virginia, and the Texas Advanced Research Program for their support.

$\dagger$ To whom correspondence should be addressed. Electronic address: cox@uvahep.phys.virginia.edu
[1] M. B. Einhorn and S. D. Ellis, Phys. Rev. D12, 2007(1975); H. Fritzsch, Phys. Lett. B67, 217(1977); M. Glück, J. F. Owens and E. Reya, Phys. Rev. D17, 2324(1978).

[2] C. H. Chang, Nucl. Phys. B172, 425(1980); E. L. Berger and D. Jones, Phys. Rev. D23, 1521(1981); R. Baier and R. Rückl, Phys. Lett. B102, 364(1981) and Zeit. Phys. C19, 251(1983).

[3] G. T. Bodwin, E. Braaten, and G. P. Lepage, Phys. Rev. D51, 1125(1995); For a recent review and a more complete list of references see also 12 .

[4] M. Beneke and I. Z. Rothstein, Phys. Rev. D54, 2005(1996); G. A. Schuler, Zeit. Phys. C71, 317(1996).

[5] T. Alexopoulos et. al., Nucl. Instr. and Meth. A376, 375(1998).

[6] L. Antoniazzi et. al., Nucl. Instr. and Meth. A360, 334(1995).

[7] T. Alexopoulos et. al., Phys. Lett. B374, 271(1996).

[8] T. Sjöstrand, CERN-TH.7112/93.

[9] CERN Applications Software Group, CERN Program Library Long Writeup W5013.

[10] C. Caso et. al., Eur. Phys. J. C3, (1998).

[11] D. A. Bauer et. al., Phys. Rev. Lett. 54, 753(1985); L. Antoniazzi et. al., Phys. Rev. D49, 543(1994).

[12] M. Beneke, The Strong Interaction, From Hadrons to Protons, edited by J. Chan, L. DePorcel, and L. Dixon (Stanford, CA, 1996), p. 549; hep-ph/9703429.

[13] S. Gupta and P. Mathews, Phys. Rev. D55, 7144(1997). and ibid. 56, 3019(1997).

[14] T. Alexopoulos et. al., Phys. Rev. D55, 3927(1997). 